\section{Anesthesia techniques for carotid endarterectomy}

\author{
DINKO TONKOVIĆ • DANIJELA BANDIĆ-PAVLOVIĆ \\ ROBERT BARONICA - TAJANA ZAH-BOGOVIĆ • SANJA SAKAN \\ ELONORA GOLUŽA ・ ŽELJKO DRVAR
}

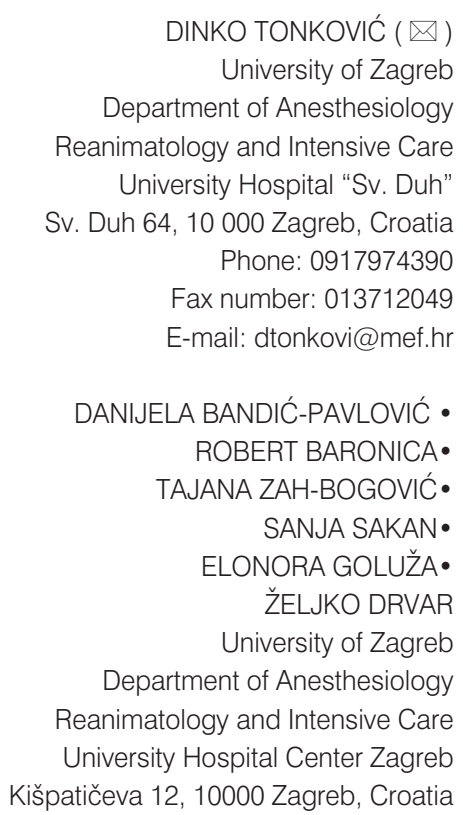

DINKO TONKOVIĆ ( $\square)$

University of Zagreb

Department of Anesthesiology

Reanimatology and Intensive Care

University Hospital "Sv. Duh"

Sv. Duh 64, 10000 Zagreb, Croatia

Phone: 0917974390

Fax number: 013712049

E-mail: dtonkovi@mef.hr

DANIJELA BANDIĆ-PAVLOVIĆ • ROBERT BARONICA •

TAJANA ZAH-BOGOVIĆ•

SANJA SAKAN•

ELONORA GOLUŽA•

ŽELJKO DRVAR

University of Zagreb

Department of Anesthesiology

Reanimatology and Intensive Care

University Hospital Center Zagreb

Kišpatičeva 12, 10000 Zagreb, Croatia

\begin{abstract}
Stroke is one of the leading causes of death in the modern countries. Mainstay treatment for stroke prevention is carotid endarterectomy (CEA). Patients scheduled for surgery often have many associate systemic illnesses that pose a risk of perioperative cardiac and neurological complications. Detailed preoperative evaluation of neurological and cardiac function with optimization of the systemic illnesses therapy is obligatory. Ideal anesthesiology technique should provide adequate analgesia, minimal stress response, optimal brain perfusion and oxygenation, optimal hemodynamic and myocardial oxygen balance while assuring calm and relaxed patients with good surgical comfort. Both regional anesthesia and general anesthesia have some advantages and drawbacks. Regarding to cerebral and myocardial ischemia and adverse outcome after CEA, especially in high risk patients, today still it is not clear which anesthesia technique is preferred for CEA. Greatest risk in the early postoperative period is new neurological deficit caused by cerebral ischemia end myocardial infarction caused with hemodynamic instability and therefore CEA patients are placed in the Intensive Care Unit for at least six or more hours where they are monitored for neurological and hemodynamic complications.
\end{abstract}

Key words: anesthesia, carotid endarterectomy

\section{Introduction}

Stroke is one of the leading causes of death in modern countries. (1) It is caused by carotid artery stenosis with atherosclerotic plaque or atheroma embolization. The mainstay of treatment for stroke prevention is carotid endarterectomy (CEA). It is indicated in symptomatic patients with carotid artery stenosis $>70 \%$, in symptomatic patients with stenosis $50-69 \%$ and in asymptomatic patients with stenosis $>$ $70 \%$ if the preoperative risk is acceptable. Although CEA is a preventive procedure, perioperative mortality is relatively high, about $5 \%$. The main risks are cerebral and myocardial ischemia. Cerebral ischemia may occur during temporary clamping of the common carotid artery (while the artery is opened for atherosclerotic plaque removal) producing hypoperfusion, if the collateral blood flow is inadequate. Prevention of cerebral ischemia is possible with shunt placement in the carotid artery.
However, surgical manipulation during shunt placement can cause embolization, damage of the vessel and also produce cerebral ischemia. Patients with carotid artery disease often have generalized atherosclerosis that pose a risk for myocardial ischemia caused by hemodynamic instability during surgery. Although local anesthesia was predominant at the beginning of CEA, general and regional techniques are better options because of patient comfort. (2) In view of the risks of cerebral and myocardial ischemia and other 
adverse outcomes after CEA, especially in high risk patients, it is still not clear today which anesthesia technique is preferred for CEA. $(3,4)$

\section{Preoperative evaluation}

Patients scheduled for CEA often have many associated systemic illnesses, like atherosclerosis, arterial hypertension and diabetes mellitus that pose a risk for perioperative cardiac and neurological complications. Detailed preoperative evaluation of neurological and cardiac function with optimization of therapy for systemic illnesses is obligatory. Neurological evaluation includes neurological exam with evaluation of prior neurological deficits and collateral cerebral circulation. (3) If the patient already suffered a stroke, it is likely that the collateral cerebral circulation is inadequate and that intraoperative cerebral ischemia, during clamping of the carotid artery, may occur. In such cases, intraoperative placement of a shunt, with the advantages of general anesthesia and control of oxygenation and hemodynamics, should be planned. Patients with preoperative hypertension and diastolic pressure higher than $110 \mathrm{mmHg}$ have more frequent neurological and cardiac complications. Perioperative normalization of blood pressure values is recommended, and patients with blood pressure values higher then $180 / 110 \mathrm{mmHg}$ should be postponed until normalization of blood pressure. Evaluation of coronary artery disease should be done according to the guidelines of the American society of cardiologists for preoperative evaluation of cardiac patients scheduled for noncardiac surgery. Diabetes, especially its late complications such as neuropathy and impairment of renal function, could cause hemodynamic instability and difficulties with perioperative volume evaluation and optimization. Hyperglycemia worsens cerebral ischemic injury and tight perioperative glycemia control is mandatory.

\section{Monitoring}

Routine hemodynamic monitoring includes electrocardiography (ECG)

Table 1. The techniques used for neurological monitoring during carotid endarterectomy.

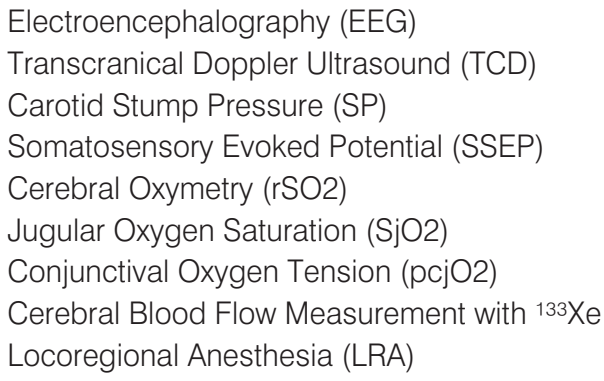

with II and V5 leads and invasive arterial pressure. Patients with impaired cardiac function should have a pulmonary artery catheter or some other type of hemodynamic monitoring such as PICCO or LIDCO. (4) Monitoring of cerebral ischemia is of utmost importance for avoiding serious neurological complications. If cerebral ischemia is detected after clamping of the carotid artery, the surgeon and anesthesiologist have several maneuvers to relieve it completely (eg. by declamping the artery, increasing arterial pressure, increasing oxygenation etc), thus avoiding any unwanted outcomes. The best way to monitor cerebral ischemia is controversial. All instrumental monitoring methods (table 1) that detect perfusion or electric activity of the brain are nonspecific. Cerebral blood flow mainly depends on vascular autoregulation and brain oxygenation and is very complex and multifactorial. To avoid cerebral ischemia during iatrogenic occlusion of the carotid artery it is crucial to correctly estimate collateral circulation and preserved cerebrovascular autoregulation, that is heavily influenced by general anesthesia.

During occlusion of the carotid artery, surgeons traditionally demand iatrogenic hypertension although only one third of postoperative cerebral ischemia is caused by hypoperfusion. (4) On the other hand embolization during surgical manipulation and shunt placement is the main cause of postoperative cerebral ischemia in two thirds of patients. Hypertension itself is not harmless. Higher levels of blood pressure increase myocardial oxygen demand and increase risk of myocardial infarction. Hypoperfusion of the brain with the shut down autoregulation done by general anesthesia can produce higher perfusion of the dilated vessels while lowering the perfusion even more in the vasoconstricted vessels producing cerebral ischemia and also producing hemorrhagia in the suddenly well perfused brain without the capability of autoregulation. Brain oxygen balance is a dynamic process influenced by many factors such as cerebral vascular anatomy, surgery and anesthesia, temperature changes, $\mathrm{pH}$ changes, blood viscosity and flow changes that interact with one another making exact monitoring of brain ischemia difficult. Thus, correct prediction of neurological complications with monitoring methods is not possible. The best method of functional brain monitoring is to keep the patient awake during regional anesthesia. Monitoring of cerebral ischemia is done with verbal communication through the assessment of patient's ability to squeeze a ball with the contra lateral arm from the operation site during the clamping of the carotid artery. (5)

\section{Anesthesia techniques}

An ideal anesthesiology technique should provide adequate analgesia, minimal stress response, optimal brain perfusion and oxygenation, optimal hemodynamic and myocardial oxygen balance while assuring calm and relaxed patients with good surgical comfort. (5) The type of anesthesia depends on the competence and pre- 
disposition of the surgeon and anesthesiologist as well as the cooperation and capability of the patients. (5) General anesthesia is recommended for patients with a history of prior cerebral strokes, if the brain collateral circulation is inadequate and for patients with distal stenosis of the carotid artery due to complicated surgical technique. (6) Patients can also refuse regional anesthesia and one of the indications for general anesthesia is an uncooperative patient. During general anesthesia, a balanced technique is used. For induction, all standard intravenous agents can be used except thiopental because of its possible negative inotropic effect. Inhalation agents are used for maintenance of anesthesia. Advantages of general anesthesia include mechanical ventilation with controlled oxygenation and carbon dioxide $\left(\mathrm{CO}_{2}\right)$ levels, controlled hemodynamics and good surgical conditions. Some anesthetic agents have a favorable effect on the oxygen balance in the brain and in the heart, theoretically preventing hypoxic injury. Protective effects are due to decreased metabolism rate, stress response and anesthetic intrinsic action. As neuroprotective agents, thiopental and propofol are especially effective. Inhalational anesthetic agents also decrease oxygen demand but on the other hand increase perfusion and intracranial pressure. Isofluran and sevorane have the best neuroprotective effect. Opioids have an indirect effect on oxygen balance by decreasing the stress response. Muscle relaxants decrease oxygen consumption in the muscles providing more oxygen to other tissues. Disadvantages of general anesthesia include inability of reliable neuromonitoring and more frequent placement of shunts which carries a greater risk for embolization. Cerebral perfusion pressure is well autoregulated regardless of the mean arterial pressure, in the range 50 - $140 \mathrm{mmHg}$, while depending on arterial perfusion pressure above and below that range. All anesthetic drugs down regulate cerebral perfusion autoregulation leading to greater correlation of cerebral perfusion pressure with arte- rial pressure. Decreased sympathetic activity during general anesthesia can produce severe hypotension especially in patients with generalized atherosclerosis and decreased autonomic response. Surgical manipulation of the carotid baroreceptors also can produce hypotension and severe bradycardia. On the other hand general anesthesia decreases brain oxygen demand by protecting the brain from ischemia during hypoperfusion. Exact, safe lower and higher levels of arterial pressure are hard to define. An additional drawback of general anesthesia is the risk of delayed emergence caused by temporary cerebral hypoperfusion and decreased metabolism of anesthetic drugs during hypotensive episodes. (6) Successful regional anesthesia demands cooperation between surgeon, anesthesiologist and the patient. Regional techniques must create a block of the cervical nerve roots from C2 to C4. Types of regional techniques include superficial and profound blocks of the cervical plexus, cervical epidural anesthesia and local anesthesia. (7) Cervical epidural anesthesia is seldom used because of possible adverse effects. Local anesthesia can lead to bad surgical conditions because of the patient's discomfort. (8) Traditionally, regional anesthesia is carried out as a combined superficial and profound block. Recently, a third type of regional anesthesia was described: an intermediate block as a sole technique. Drugs for regional anesthesia include bupivacaine, chirocaine and ropivacaine in the concentrations form 0,375 to $0,5 \%$ with $2 \mathrm{ml}$ of epinephrine in concentration 1:200 000. Superficial cervical block is a simple procedure with a low incidence of complications. (9) Patients are lying flat with the head turned away from the operation side. For anxiety relief a bolus dose of opioids can be used. After sterile washing and preparing of the operative field, the posterior border of the sternocleidomastoid muscle is identified asking the patients to lift the head while looking to the opposite side. Then $5 \mathrm{ml}$ of a local anesthetic are injected with a 22 gauge needle at the height of the cricoid cartilage directly perpendicular to the skin and $5 \mathrm{ml}$ more superiorly and inferiorly along the border of the sternocleidomastoid muscle. Depth of injected local anesthetics is $1,5 \mathrm{~cm}$ subcutaneously. A superficial block alone is seldom sufficient for CEA so it is often complemented with a profound block. Profound cervical block is more demanding with the possibility of serious complications but assures better anesthesia quality. Patient position is the same as for the superficial block. Before sterile drapes are placed, a line is drawn along the posterior border of the sternocleidomastoid muscle that connects the Chassaignac tuberculum of the transverse process of the $\mathrm{C} 2$ vertebrae and the mastoid. After that, another line is drawn $1 \mathrm{~cm}$ posterior and parallel with the first one. (10) The cervical plexus is blocked when the nerve roots pass along the transverse process. Identification of the transverse process of the $\mathrm{C} 2-\mathrm{C} 4$ vertebrae is made $1-2 \mathrm{~cm}$ below the mastoid process with a 1-1, $5 \mathrm{~cm}$ distance between them along the second line. Local anesthetic is administered at the identified places with a 22 gauge, $5 \mathrm{~cm}$ long needle to a depth of $3 \mathrm{~cm}$. Patients should feel paresthesia with the injection after which $10-12 \mathrm{ml}$ of a local anesthetic is injected at the C2, C3 and C4 levels or only at the C2 and C3 levels. Profound cervical block blocks n.phrenicus so bilateral block is contraindicated. Complications of the profound block include adherent intravascular injection. The risk is greater in superficial and intermediate block and complication that can occur is high spinal anesthesia, with cardiovascular collapse and respiratory insufficiency. Intermediate block is the same as superficial block but the depth of needle insertion is about 2-2, $5 \mathrm{~cm}$ and a larger volume of local anesthetic is injected, about $10 \mathrm{ml}$ every time. Intermediate block has very good anesthetic quality with a low incidence of complications. Seldom, regional cervical blocks are supplemented with local anesthetic, infiltrated between the jugulum and the thyroid cartilage and superficially below the mandible to pre- 
vent sensation during skin retraction intraoperately due to crossover innervation of that area with the contralateral cervical plexus.

Advantages of the regional anesthesia technique are functional neuromonitoring and good hemodynamic stability. (11) During regional anesthesia, autoregulation of brain circulation is maintained providing physiological distribution of blood flow during hypoperfusion periods that preserve oxygen balance. Disadvantages of regional anesthesia include possibilities of local anesthetic adverse effects, difficult airway control in the case of unsuspected cardiorespiratory arrest during clamping of the carotid artery, lack of the anesthetic's neuroprotective effects and surgeon's discomfort. A few small studies have shown that patients with regional anesthesia scheduled for CEA have a lower incidence of cerebral and myocardial ischemia with shorter hospital stay and lower costs. (12) A recent metaanalysis, that compared general and regional anesthesia for CEA, found no statistically significant difference between regional and general in the number of perioperative neurological and myocardial complications and death during the first 30 postoperative days. However, in the same study the risk of stroke seemed higher during general anaesthesia than under regional anaesthesia in patients with contralateral-carotid occlusion although this was not statistically significant. (12) Those findings could be explained by better neurological monitoring, avoidance of routine shunt placement, and better hemodynamic stability during regional anesthesia. Clear benefit of one over the other anesthesia technique may be lacking because of modern monitored general anesthesia, the use of potent vasoactive drugs and maybe from excluding high risk patients in whom differences may be more obvious.

\section{Postoperative treatment}

The greatest risk in the early postoperative period is that of a new neurological deficit caused by cerebral ischemia or myocardial infarction associated with hemodynamic instability. Postoperative hypertension may be a result of carotid baroreceptor trauma and it is more prominent in patients with preoperative hypertension. Hyperperfusion of the brain after CEA on the operated side could lead to hyperperfusion syndro- me. Hyperperfusion syndrome could be severe leading to serious complications including: anxiety, headache, convulsion, and intracranial hemorrhage with coma and death. Risk of hyperperfusion is especially high in patients with hypertension and with no preserved cerebrovascular autoregulation after general anesthesia. Some patients develop postoperative hypotension that is caused by increased perfusion of the brain with reflex hypotension and bradycardia. Hypotension also can be caused by absorption of large doses of local anesthetics. After CEA patients are placed in the Intensive Care Unit (ICU) for 6 or more hours where they are monitored for neurological and hemodynamic complications. Recommended values of postoperative blood pressure are up to $30 \%$ lower or higher than preoperative values. Postoperative analgesia is maintained with opioids and paracetamol while hemodynamic stability is maintained with a continuous infusion of vasoactive drugs. Patients that undergo regional anesthesia have better hemodynamic stability with better postoperative analgesia. Hemodynamic disturbances can occur up from 4 to 24 hours postoperatively after the patients are sent to the wards.

\section{REFERENCES}

1. Učestalost kroničnih nezaraznih bolesti. Hrvatski zavod za javno zdravstvo. Služba za epidemiologiju masovnih kroničnih bolesti (homepage on the internet). (Updated 2009; cited August 16 2011) Available from: http://www.hzjz.hr/epidemiologija/kron bol.htm

2. Howell SJ. Carotid endarterectomy. BJA 2007;99 (1):119-31.

3. Meitzner MC, Skurnowicz JA, Mitchell A. A literature review on anesthetic practice for carotid endarterectomy surgery based on cost, hemodynamic stability, and neurologic status. ANNA Jour 2007;75 (3):193-7.

4. Shun-ichi N, Kiyoshi K, Hiroshi A. Hemodynamic stability under general anesthesia in carotid artery stenting. Radiat Med 2005;23(6):427-31.

5. Rossel T, Litz RJ, Heller AR, Koch T. Anaesthesia for carotid artery surgery. Is there a gold standard? Anaesthesist 2008;57(2):113

6. Farhoomand L, Berger JM, Lehfeldt S. Controversies in anesthesia for carotid endarterectomy: general versus regional anesthesia. Semin Anesth Perioperat Med Pain 2004;23(3):244-7.

7. Stoneham MD, Knighton JD. Regional anaesthesia for carotid endarterectomy. BJA 1999;82(6):910-19.

8. Gough MJ. Local versus GA for carotid endarterectomy: Improving the gold standard?:Invited commentary. Eur J Vasc Endovasc Surg 2008;36:150-1

9. Ivanec Ž, Mazul-Sunko B, Lovričević I. Superficial versus combined (deep and superficial) cervical plexus block for carotid endarterectomy. Acta Clin Croat 2008;47:81-6.

10. Superficial cervical plexus block and deep cervical plexus block. The New York School of Regional Anesthesia Official. (Updated January 2011; cited August 2012) Available from: http://www.nysora.com

11. Lutz HJ, Michael R, Gahl B, Savolainen H. Local versus general anaesthesia for carotid endarterectomy-Improving the gold standard?. Eur J Endovasc Surg 2008; 36:145-9.

12. Guay J. Regional anesthesia for carotid surgery. Curr Opin Anaesthesiol 2008;21:638-44. 